\title{
Taste aversions and acute methyl mercury poisoning in rats*
}

\section{J. JAY BRAUN and DANIEL R. SNYDER \\ Yale University, New Haven, Conn. 06510}

Rats injected with methyl mercuric chloride $(5 \mathrm{mg} / \mathrm{kg})$ following exposure to the taste of saccharin rejected saccharin on subsequent days to a significantly greater extent than either rats poisoned following plain water or rats injected with normal saline following saccharin exposure. Intraperitoneal injections caused nonspecific reduction of fluid intake in addition to the specific rejection of saccharin, and intravenous injections were not accompained by nonspecific effects. The present study adds methyl mercuric chloride to the expanding list of substances that are effective unconditioned aversive stimuli for learned taste aversions and testifies to the sensitivity and potential utility of the taste aversion technique for studying the effects of acute exposure to this chemical.

Aversions to distinctively flavored substances can be produced readily in rats and other animals simply by accompanying presentation of the flavor with the administration of a poison (Chitty, 1954), a nausea-producing drug, or irradiation (e.g., Garcia \& Koelling, 1967; Smith, 1971). This effect has been explored in detail by Garcia and colleagues (Garcia \& Koelling, 1967; Garcia, Kimeldorf \& Hunt, 1968), who, along with others (e.g., Revusky, 1968; Lorden Kenfield, $\&$ Braun, 1970) have argued that it is an associative learning phenomenon.

The present experiment uses the taste-aversion paradigm to test the effectiveness of methyl mercuric chloride as an unconditioned aversive stimulus. By comparing the effects of two routes of administration, intraperitoneal (IP) and intravenous (IV), it was hoped that some indication of the mechanism of action of the chemical in this situation might be revealed. The clinical signs of mercury poisoning reported in the literature generally attest to highly selective irreversible neuropathology when ingested in toxic amounts (e.g., Hunter, Bomford, \& Russell, 1940; Morikawa, 1961). Therefore, a sensitive behavioral indicator of mercury poisoning might be useful for the investigation of short-term, reversible, or low-level methyl mercury toxicity. Accordingly, the present study investigated the sequelae of acute IP and IV doses of methyl mercuric chloride in rats following exposure to a novel taste cue (saccharin).

\section{METHODS}

The methyl mercuric chloride, obtained from Alfa Inorganics, was slowly dissolved in distilled water $(5 \mathrm{mg} / \mathrm{cc})$ and stored in sealed, multiple-dose injection vials.

In pilot studies using male Strain DA rats from our colony $(212-284 \mathrm{~g})$, it was found that IP doses of $20 \mathrm{mg} / \mathrm{kg}$ and

*This research was partially supported by USPHS Research Grants NS 08658 to J.J. Braun and 2-P06-RR00393 and 5-S01-RR05358 to A.M Jonas. We are grateful to Edward J. Gralla and Albert $M$. Jonas for support. Request reprints from J. Jay Braun, Department of Psychology, Arizona State University, Tempe, Arizona 85281.
$10 \mathrm{mg} / \mathrm{kg}$ were lethal for all rats injected $(\mathrm{N}=4)$ within 5 to $12 \mathrm{~h}$. Marked symptoms of poisoning appeared within $30 \mathrm{~min}$ in the following order: (1) crouching in rear of cage, eyes partially closed; (2) sluggish motor responses when prodded; (3) recumbent on side with hyperventilation; (4) rear leg extension with twisting and stretching of hind quarters; and (5) gasping with colonic limb twitching. Intravenous injection of $20 \mathrm{mg} / \mathrm{kg}$ into, the tail vein had much less pronounced effects: quiescence and crouching were evident but the gasping recumbancy was absent. Two rats, administered $5 \mathrm{mg} / \mathrm{kg}$ IP, displayed some very mild initial symptoms which had disappeared by the following day, and no observable symptoms were produced by equivalent IV injections. Pathological examination of the IP-dosed rats that died revealed both macroscopic and microscopic signs of acute chemical peritonitis (also described by Swensson, 1952). Neither the behavioral signs nor the tissue response of peritonitis were observed following equivalent IV doses.

Next, 41 male Strain DA rats, weighing 298 to $368 \mathrm{~g}$, were accustomed to a water drinking schedule of $30 \mathrm{~min}$ free drinking per day from two calibrated $50-\mathrm{ml}$ centrifuge tubes of tap water mounted on their cage fronts once every $23 \frac{1}{2} \mathrm{~h}$ for 20 to 40 days. The rats were then assigned to four groups on a random basis and, on the training day (Day 0), both drinking bottles were filled with either water (W), as usual, or with $1.5 \mathrm{~g} / 1$ sodium saccharin (S) solution. The Day 0 drinking session was followed immediately by either an IP injection (Groups SP and WP) or an intravenous injection (Groups SV and WV) of $5 \mathrm{mg} / \mathrm{kg}$ methyl mercury; rats in the control groups received, following exposure to saccharin, either an IP injection of physiological saline $(N=7)$ equivalent in volume to the methyl mercury injection (approximately $0.23 \mathrm{cc}$ ), or tail venipuncture and attendant trauma $(\mathrm{N}=6)$. These groups served as a control for generalized trauma effects of the injection procedure, and since neither procedure without the drug appeared to affect subsequent consummatory behavior and the two subgroups did not appear to differ, they are considered as one below (Group C). Rats in the IV-injected groups for which part of the injection missed the tail vein were discarded, and this resulted in smaller group sizes than intended.

On Days 1-4, each rat was offered two bottles, one containing saccharin and the other water, at the regular watering time (the relative positions of the bottles were alternated daily), and the amount of each solution drunk (in milliliters) by each rat was recorded.

\section{RESULTS}

The results are summarized in Table 1 , which presents for each group the average total fluid volume consumed and the average proportion of this volume representing saccharin intake. The Kruskal-Wallis one-way analysis of variance revealed significant differences among groups within each measurement category $(p<.001)$. The Mann-Whitney $U$ test (two-tailed) was used for the paired comparisons reported below (Siegel, 1956).

Rats poisoned with methyl mercury following exposure to the novel saccharin solution drank a significantly smaller proportion of saccharin than did the nonpoisoned group (compare SP to $\mathrm{C}$ and compare $\mathrm{SV}$ to $C$, both $(p<.002)$, and they also drank less saccharin than the groups poisoned following regular tap water (compare SV to WV, $p<.02$; and SP to WP, $p<.002$ ). The suppressed comsumption of saccharine in the two experimental groups appears, therefore, to be related 
Table 1

Median/Mean Total Fluid Volume (Milliliters) Consumed Over Days 1-4 and the Proportion of That Volume Representing Accumulated Saccharin Intake*

\begin{tabular}{|c|c|c|c|}
\hline Group & $\mathbf{N}$ & $\begin{array}{c}\text { Total Fluid } \\
\text { Volume Summed } \\
\text { Over } 4 \text { Days }\end{array}$ & $\begin{array}{l}\text { Saccharin } \\
\text { Proportion }\end{array}$ \\
\hline $\mathrm{C}$ & 13 & $78.0 / 80.8$ & $.60 / .61$ \\
\hline WV & 4 & $78.5 / 78.3$ & $.61 / .61$ \\
\hline SV & 6 & $78.0 / 81.5$ & $.35 / .35$ \\
\hline WP & 6 & $66.5 / 64.0$ & $.50 / .51$ \\
\hline SP & 12 & $58.5 / 59.0$ & $.19 / .20$ \\
\hline
\end{tabular}

*In the group identification, $S$ (sodium saccharin) and $W$ (water) identify the drinking solutions that were exclusively presented on the training day (Day 0), while $P$ (intraperitoneal) and $V$ (intravenous) represent the injection route used for the administration of methyl mercuric chloride. Group $C$, the control group, drank saccharin on Day 0, was subjected to the injection procedures, but was not injected with methyl mercury.

principally to the prior pairing of saccharin and methyl mercury injection, since neither variable alone had such a pronounced effect.

Intraperitoneal injection produced nonspecific effects not observed following IV injection. These included lower total fluid intake $(p<.002)$ and a tendency for the group injected following exposure to tap water (Group WP) to drink less fluid $(p<.03)$ and a lower proportion of saccharin $(p<.06)$ than the nondrugged Control Group C. In addition, the IP-injected saccharin group (SP) displayed a greater aversion to the saccharin cue $(p=.02)$ than the IV-injected group (SV). Since this latter statistic is based on a proportional measure of total fluid consumed, it does not simply reflect the lesser tendency of Group SP to drink fluids of any kind.

Intravenous injection markedly suppressed saccharin intake in Group SV, but nonspecific effects were not observed from this route of administration: Fluid consumption remained normal, and Group WV maintained saccharin consumption following mercury treatment paired with water.

After the experiment, rats from each group were perfused intracardially with physiological saline and $10 \%$ formal-saline. The brains were removed, embedded in paraffin, sectioned at 7-10 micra, and alternate sections were mounted for Hemotox-Eosin or Kluever staining. Histological examination with particular attention to the internal granular layer of the cerebellum and the primary visual cortex (Hunter et al, 1940; Morikawa, 1961; Berlin et al, 1963) did not reveal obvious morphological neuropathological changes.

\section{DISCUSSION}

One of the present barriers to understanding the nature of learned taste aversions is the lack of specification of the receptors and related neural pathways of the unconditioned stimulus. Although the procedures for producing the learned aversions can be operationally specified, the physiological consequences of these procedures critical for the development of an association between a flavor cue and toxicosis, cannot be so specified. The peripheral consequences of administering organic mercury compounds to animals are peritonitis (if administered IP-see Method, Paragraph 2) and relatively rapid accumulation of the compound in the viscera (Berlin \& Ullberg, 1963). Centrally, autoradiographic studies indicate CNS uptake within minutes of a single IV injection, but at a rate that is much slower than peripheral uptake. Also, the area postrema, described as a "vomiting center" in several species other than rats (see Brizzee, 1956), along with the cerebellum, appears to be a primary central deposition area for methyl mercury (Berlin \& Ullberg, 1963).

The present study demonstrates that acute subclinical dosages of methyl mercuric chloride have distinct aversive consequences for rats, as measured by taste aversion learning criteria. When the chemical was administered IV, its effects were obvious only through the associative measure (relative reduction of saccharin intake in Group SV as compared to Group WV) employed in this study. This supports Berger's (1972) observation that conditioned taste aversions can occur without overt signs of toxicosis. Intraperitoneal injections of the chemical produced a greater subsequent rejection of the taste cue than did IV injections, as well as a nonspecific reduction in the total fluid volume consumed, and the control group injected IP following plain water (Group WP) displayed a selective aversion to saccharin as compared to Group C, which was not injected with mercury.

It is tempting to regard the specific associative effects of the IV and IP injections as produced by a central action of the drug, and the nonspecific effects of the IP injections as produced by peripheral irritative visceral input from the peritonitus associated with IP injections. Any such irritative properties resulting from IV injections presumably would be greatly diminished by blood dilution and by the rapid binding of methyl mercury to the erythrocytes. However, since the present experiment does not completely exclude the possibility of residual irritative visceral stimulation from the IV injections, a strong case for the above interpretation cannot be made at this time.

\section{REFERENCES}

Berger, B. Conditioning of food aversions by injections of psychoactive drugs. Journal of Comparative \& Physiological Psychology, 1972, 81, 21-26.

Berlin, M., \& Ullberg, S. Accumulation and retention of mercury in the mouse. Archives of Environmental Health, 1963, 6, 589-616.

Brizzee, $K$. R. Effect of localized brain stem lesions and supradiaphragmatic vagotomy on $X$-irradiation emesis in the supradiaphragmatic vagotomy on X-irradiation emesis in the
monkey. American Journal of Physiology, 1956, 187, monkey.

Chitty, D. (Ed.) Control of rats and mice, Volume 5, London: Oxford University Press, 1967.

Garcia, J. Kimelldorf, D. J., \& Hunt, E. L. The use of ionizing radiation as a motivating stimulus. Psychological Review, $1961,68,383-395$.

Garcia, J., \& Koelling, R. A. A comparison of aversions induced by $X$-rays, toxins, and drugs in the rat. Radiation Research Supplement, 1967, 7, 439-450.

Hunter, D., Bomford, R., \& Russel, D. S. Poisoning by methyl mercury compounds. Quarterly Journal of Medicine, 1940, 9 193-214.

Lorden, J. R., Kenfield, M., \& Braun, J. J. Response suppression to odors paired with toxicosis. Learning \& Motivation, 1970, 1, 391-400.

Morikawa, N. Pathological studies on organic mercury poisoning. Part I. Experimental organic mercury poisoning in cats and its relation to the causative agent of Minamata disease. Kumamoto Medical Journal, 1961, 14, 71-86.

Revusky, S. H. Aversion to sucrose produced by contigent X-irradiation: Temporal and dosage parameters. Journal of Comparative \& Psysiological Psychology, 1968, 65, 17-22.

Siegel, S. Nonparametric statistics for the bahavioral sciences. New York: McGraw-Hill, 1956.

Smith J. R. Radiation: Its detection and its effects on taste preferences. In E. Stellar and J. M. Sprague (Eds.), Progress in physiological psychology. Vol 4. New York: Academic Press, 1971.

Swenson, A. Investigations on the toxicity of some organic mercury compounds which are used as seed disinfectants. Acta Medica Scandinavica, 1952, 365-384.

(Received for publication March 19, 1973.) 